\title{
Pomelo Peel-Inspired 3D-Printed Porous Structure for Efficient Absorption of Compressive Strain Energy
}

\author{
Baisong Yang ${ }^{1} \cdot$ Wenhui Chen ${ }^{1} \cdot$ Renlong Xin ${ }^{2} \cdot$ Xiaohong Zhou $^{1} \cdot$ Di Tan $^{1} \cdot$ Chuan Ding ${ }^{1} \cdot$ You Wu ${ }^{1} \cdot$ Liang Yin $^{1}$. \\ Chuyang Chen ${ }^{1}$. Shan Wang ${ }^{3}$. Zhenglei Yu ${ }^{2}$. Jonathan T. Pham ${ }^{4}$. Sheng Liu ${ }^{1} \cdot$ Yifeng Lei $^{1} \cdot$ Longjian Xue $^{1}$ (i)
}

Received: 13 September 2021 / Revised: 7 December 2021 / Accepted: 14 December 2021 / Published online: 4 January 2022

(c) The Author(s) 2021

\begin{abstract}
The porous structure in pomelo peel is believed to be responsible for the protection of its fruit from damage during the free falling from a tree. The quantitative understanding of the relationship between the deformation behavior and the porous structure could pave the way for the design of porous structures for efficient energy absorption. Here, a universal feature of pore distribution in pomelo peels along the radial direction is extracted from three varieties of pomelos, which shows strong correlation to the deformation behavior of the peels under compression. Guided by the porous design found in pomelo peels, porous polyether-ether-ketone (PEEK) cube is additively manufactured and possesses the highest ability to absorb energy during compression as compared to the non-pomelo-inspired geometries, which is further confirmed by the finite element simulation. The nature-optimized porous structure revealed here could guide the design of lightweight and high-energydissipating materials/devices.
\end{abstract}

Keywords Bionic design $\cdot$ Pomelo peel $\cdot$ Porous structure $\cdot 3 \mathrm{D}$ printing $\cdot$ Energy absorption

\section{Introduction}

Porous structures are abundant in nature and play an essential role in the adaptation of organisms to their living environments [1-3]. For example, the thick pomelo peel can dissipate energy up to an impressive $\sim 98 \mathrm{~J}$, allowing the fruit to withstand a deceleration force of several kilonewtons

Baisong Yang and Wenhui Chen contributed equally to this work.

Yifeng Lei

yifenglei@whu.edu.cn

$\bowtie$ Longjian Xue

xuelongjian@whu.edu.cn

1 School of Power and Mechanical Engineering, The Institute of Technological Sciences, Wuhan University, South Donghu Road 8, Wuhan 430072, Hubei, China

2 State Key Laboratory of Automotive Simulation and Control and Key Lab of Bionic Engineering, Ministry of Education, Jilin University, Changchun 130022, China

3 School of Materials Science and Engineering, Wuhan University of Technology, Wuhan 430070, China

4 Department of Chemical and Materials Engineering, University of Kentucky, Lexington, KY 40506, USA without visible damage $[4,5]$. The porous structure in pomelo peels is considered to be responsible for the ability of energy absorption [5-10]. However, a quantitative understanding of how the porous structure enables the high energy absorption across different pomelo varieties remains missing. Quantitative insight into the relationship between the porous structure of pomelo peels and their deformation under pressure could guide us in the design of lightweight, porous materials not only for energy absorption, but also for biomaterial engineering, health care, clean energy, and so on $[11,12]$.

Pioneering effects have been carried out to understand the porous structure in pomelo peels. For example, Seidel et al. [5] investigated the distribution of cell wall, cell lumen and intercellular space by light microscopy and gave an estimation that intercellular space increases from 30 to $80 \%$ from 1 to $4 \mathrm{~mm}$ away from the outermost layer (exocarp). By sectioning, Thielen et al. [9] reported the distribution of cell number over the radial thickness of a peel in the pomelo and found the lowest and highest cell density at the locations of $66.7 \%$ and 95.2\% away from the juicy pulp, respectively. Bührig-Polaczek et al. [4] proposed that fluid-filled struts and a density gradient in the middle of the peel (mesocarp) could be the key factor for the uniform collapse of the peel under quasi-static 
compression. The microstructure-related mechanical properties of pomelo peels in response to compressive loading was in situ investigated by X-ray tomographic imaging technique, suggesting a nearly linear morphology-mechanics relationship [13]. Furthermore, the water in the peel also has a strong influence on energy dissipation capacity that the removal of water from a fresh peel strongly reduces its relative energy dissipation capacity [6]. While these antecedent case studies provide insight about pomelo peels, a quantitative characterization across multiple varieties of the porous peel remains elusive.

On the other hand, some attempts have been carried out to realize enhanced mechanical properties $[4,8,14$, 15] of new materials by incorporating features inspired by pomelo peels. Using polymeric foam as the template, Bührig-Polaczek et al. [4] succeeded in the fabrication of ceramic fiber-reinforced porous alloy structures to mimic the functions of vascular bundles in pomelo peels. Fiber reinforcements parallel to the loading direction resulted in an increase in the compression strength and stiffness of up to $30 \%$. Inspired by pomelo peel, the incorporation of structural hierarchy into the honeycomb geometry suggested that the improved crushing resistance and energy absorption properties of the honeycomb geometry can be realized by the increase of structural hierarchy and variation of geometric dimensions [14]. While these researches incorporated some features inspired by pomelo peel and demonstrated the merits of these features, however, few studies have incorporated the most basic feature of pomelo peels, the porous design of the pomelo peel. We assume this may be partially due to the lack of quantitative understanding of the porous structures in varieties of pomelo peels.

Herein, the deformation behavior of the peels in three varieties of pomelos are carefully examined and are related with the quantitatively characterized pore structures in the peels. Similar size, density and porosity of pores along the thickness direction are found in all the three varieties, illustrating a universal porous design of pomelo peels, though the location of maximum porosity along the peel thickness varies. The spatial distribution of pores along the peel thickness agrees very well with the quasi-static compression process of the peel. Guided by the universal feature found in pomelo peels, porous polyether-ether-ketone (PEEK) cubes are additively manufactured and studied. The compression behavior and specific energy absorption (SEA) of these PEEK cubes illustrate the merit of the pomelo peel-inspired porous design. The work here not only provides a quantitative understanding of pomelo peels, but also offers a guiding principle for the design of lightweight, high strength and high energy absorption materials for potential applications in safety protection, tissue engineering, and clean energies.

\section{Materials and Methods}

\subsection{Materials}

Three varieties of pomelos (Citrus grandis L. Osbeck) were purchased from the local fruit market (Fig. 1a). The pomelos came from three places with a latitude difference of $6^{\circ}$ (Figs. 1). The first variety of pomelo (Pingshan Yu; originates from Fujian province, China) has a yellow exocarp (the outmost layer) and a red pulp, referred to as PSY in the following text. The second variety (Shatian $\mathrm{Yu}$; originates from Guangxi province, China) is referred to as STY, possessing a yellow exocarp and white pulp. The third variety (Ta-Koi), abbreviated as TK, originates from Thailand and has a green exocarp and pink pulp. For all the three varieties, between the exocarp layer and the pulp is a thick white layer called mesocarp. Three cylindrical blocks of pomelo peel with a diameter of $14.3 \mathrm{~mm}$ were sampled from the top, middle and bottom parts of each variety of pomelos (Fig. 1a). Three fruits of each variety were used. Therefore, 27 cylinder blocks of peels were collected from each variety of pomelo.

\subsection{Component Characterization}

Fresh peel block with mass of $\mathrm{w}_{1}$ was weighed by a balance (ME204, METTLER TOLEDO, Switzerland) and weighed again after drying in a vacuum oven at $60^{\circ} \mathrm{C}$ for $24 \mathrm{~h}$. The mass of dried sample was noted as $\mathrm{w}_{2}$. The water content was calculated by $\left(w_{1}-w_{2}\right) / w_{1}$. The dried peel block was treated in an aqueous solution of $2 \mathrm{wt} \% \mathrm{NaClO}_{2}(\mathrm{pH}=4.6)$ at $105{ }^{\circ} \mathrm{C}$ for $8 \mathrm{~h}$ to remove the lignin [16]. After a thorough washing in the mixture of ethanol and water $(v / v=1: 1)$, the sample was further treated in an $8 \mathrm{wt} \% \mathrm{NaOH}$ solution at $80{ }^{\circ} \mathrm{C}$ for $6 \mathrm{~h}$ to remove the hemicellulose [16]. Upon the second washing in the mixture of ethanol and water $(v / v=1: 1)$ and drying in a vacuum oven at $60^{\circ} \mathrm{C}$ for $24 \mathrm{~h}$, the mass of the sample was weighed as $w_{3}$. The cellulose content is then calculated by $w_{3} / w_{1}$. At least three blocks of peels from each part (top, middle and bottom parts) of a fruit were collected for measuring. Three pomelos of each variety were used.

\subsection{Mechanical Test}

Compression test of fresh pomelo peel was carried out on a universal testing machine (BOSE 3320, America) at a compression speed of $0.02 \mathrm{~mm} / \mathrm{s}$ and up to a compression strain of $65 \%$. Cyclic compression test was done at a speed of $0.04 \mathrm{~mm} / \mathrm{s}$ for loading and unloading with a compression strain up to $40 \%$. Between two successive cycles was 

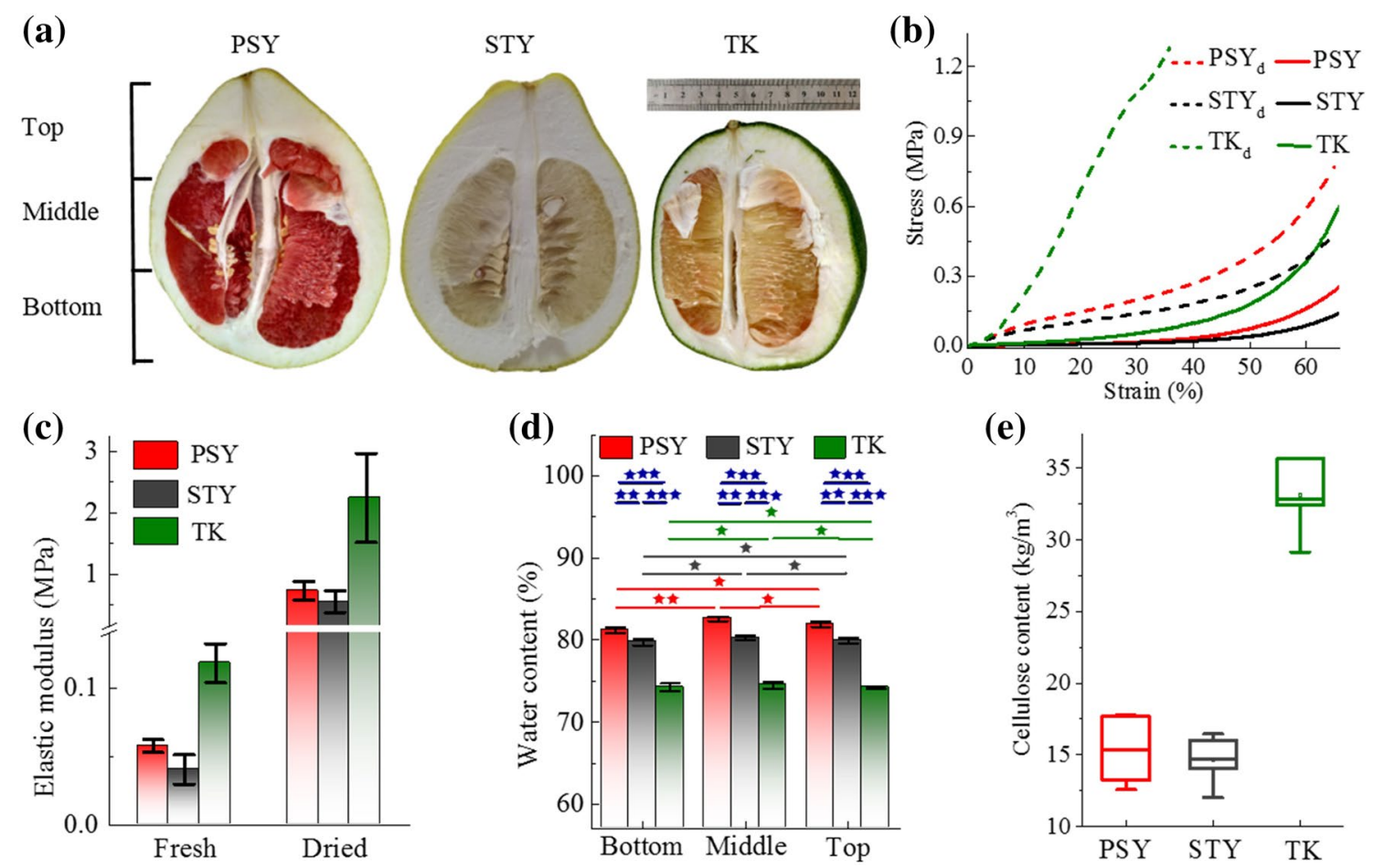

Fig. 1 Morphology and mechanical properties of pomelo peels. a Longitudinal sections of Pingshan Yu (PSY), Shatian Yu (STY) and Ta-Koi (TK). Every fruit is equally divided into three parts: top, middle and bottom parts. b Compressive stress-strain curves of fresh (PSY, STY and TK) and freeze-dried $\left(\mathrm{PSY}_{d}, \mathrm{STY}_{d}\right.$ and $\mathrm{TK}_{d}$ ) pomelo peels. c-e Elastic modulus (c), water content (d) and cellulose con-

an interval of $30 \mathrm{~s}$. Prior to the compression tests, a thin white background (TS7, TAMIYA, Japan) was sprayed on the sample surface by moving the spray can (TS7, TAMIYA, Japan) across the sample quickly, to make the white background as thin as possible; afterward, the black dots were sprayed with another spray can (TS7, TAMIYA, Japan) upward such that scattered black droplets fell onto the white background randomly. The deformation of the sample was recorded by a video camera of Matsushita, DC-GH5, Japan. Snapshots were captured in chronological order (1 picture/s) and digital image correlation (DIC) was then extracted through GOM Correlate. The compression tests of PEEK samples were carried out on INSTRON 5969, (Instron, America) with a speed of $0.02 \mathrm{~mm} / \mathrm{s}$ and a compression strain up to $70 \%$. Three peel blocks from each part of a fruit and four pomelos of each variety were used. Thus, 36 samples of each variety were collected for measurement.

\subsection{Morphology and Porosity Characterization}

Immediately after the sampling, the fresh peel blocks were filled with embedding medium, Tissue-Tek OCT ${ }^{\mathrm{TM}}$ (4853), tent (e) of pomelo peels. d One-way analysis of variance (ANOVA) test, $* p>0.05, * * 0.001<p<0.05, * * * p \leq 0.001$. The box plot in (e) is shown with median (horizontal center line), average (center point), interquartile range (IQR) (box edges), and $1.5 \times \mathrm{IQR}$ (whiskers), respectively. Values are means \pm s.d

allowed to rest for about $1 \mathrm{~h}$ and then frozen at $-20^{\circ} \mathrm{C}$ for $30 \mathrm{~min}$. The frozen peel block was evenly cut into six layers using a freezing microtome (LEICA CM 1950, Leica, Germany) at $-20^{\circ} \mathrm{C}$ and one slice with thickness of $100 \mu \mathrm{m}$ from the same location in each layer was collected. Thus, 162 slices were collected for each variety of pomelo. After washing away the embedding medium in deionized water, the collected slices were quenched in liquid nitrogen and freeze dried in a freeze dryer (FD5-2.5, GOLD-SIM, America) for $12 \mathrm{~h}$.

The collected slices were characterized by Scanning Electron Microscope (SEM, MIRA 3 LMH, Tescan AG, Czech Republic). Randomly selected no less than three locations on each slice were measured. The obtained SEM images were then processed by software ImageJ (version 1.52a) (Figs. 2). Since the pomelo peel is composed of cell walls, lumens surrounded by cell walls and intercellular spaces surrounded by lumens $[9,10]$, only the intercellular spaces (blank area in SEM images) are considered as pores in peels. Considering all the blank areas as ideal circles, the radius of a circle $\left(R_{\mathrm{i}}\right)$ was calculated based on its area $\left(S_{\mathrm{i}}\right)$ : 
$R_{\mathrm{i}}=\sqrt{\frac{S_{\mathrm{i}}}{\pi}}$

$R_{\mathrm{i}}$ is considered as the pore radius $(R)$ in pomelo peels. Pore density $(\rho)$ is defined as the number of pores $(n)$, which is counted by the software, within the detected area $(S)$ with unit of $\mathrm{mm}^{-2}$ :

$\rho=\frac{n}{S}$.

Considering all the pores, the porosity $(\sigma)$ is defined as follows:

$\sigma=\frac{\sum_{1}^{n} S_{\mathrm{i}}}{S} \times 100 \%$

Nonlinear least squares was used for data fitting to reveal the dependence of pore geometries on the relative position (RP) along the direction of peel thickness.

\subsection{Additive Manufacturing of Pomelo Peel-Inspired Structures}

The PEEK filament with tensile strength of $100 \mathrm{MPa}$ (ISO 527) and compressive strength of $125 \mathrm{MPa}$ (ISO 604) was purchased from Jilin Joinature Polymer Co., Ltd.

Porous cubic samples of PEEK were additively manufactured by a 3D printer equipped with a nozzle with diameter of $0.25 \mathrm{~mm}$ (FUNMAT HT, INTAMSYS TECHNOLOGY Co., Ltd, China). The thickness of the printing layer was $0.1 \mathrm{~mm}$ and the printing speed was $30 \mathrm{~mm} / \mathrm{s}$. The temperatures of the nozzle and the printing chamber were 410 and $90{ }^{\circ} \mathrm{C}$, respectively.

\subsection{Numerical Simulation of Pomelo Peel-Inspired Structures}

The nonlinear explicit finite element program LS-DYNA is used to simulate the quasi-static compression process of the four structures. MAT_PIECEWISE_LINEAR_PLASTICITY is used as the type of material model. Tetra fournode solid element is used for the structures and Tria3 shell element is used for the surface. Furthermore, the stiffnesstype hourglass control is implemented to get rid of the zero energy mode generally caused by the reduced integration of elements. An automatic surface-to-surface contact is applied to simulate the contact interface between the porous structure and the two rigid plates. Automatic single-surface contact is used to avoid material penetration and crossover. The static friction coefficient and dynamic friction coefficient between the contact surfaces are 0.3. The mechanical properties of PEEK are as follows: Young's modulus $E=900 \mathrm{MPa}$, Poisson's ratio $=0.28$, density $\rho=1.265 \mathrm{~g} / \mathrm{cm}^{3}$, yield stress $\sigma=80 \mathrm{MPa}$, ultimate stress $\sigma_{\mathrm{m}}=94.5 \mathrm{MPa}$. Convergence tests were carried out and $0.6 \mathrm{~mm}$ was chosen as the mesh size.

\section{Results and Discussion}

\subsection{Deformation Behavior Under Compression}

The mechanical properties of the fresh pomelo peels were quantified by compression tests and compared with the freeze-dried ones (Fig. 1b). During the compression of fresh and dehydrated pomelo peels, the linear elastic regime is followed by nonlinear behavior with no noticeable plateau that can be clearly identified [17]. As a result, the stress in both fresh and dehydrated pomelo peels does not change abruptly during compression, indicating a mild and steady deformation of the peels under pressure. The elastic moduli of fresh PSY $(58 \mathrm{kPa} \pm 4.7 \mathrm{kPa})$ and STY $(40 \mathrm{kPa} \pm 10.7 \mathrm{kPa})$ are relatively close to each other (Fig. 1c), while the fresh TK $(118 \mathrm{kPa} \pm 14.2 \mathrm{kPa})$ is much larger. Upon freeze drying, the elastic moduli for PSY, STY and TK increased to $0.73 \mathrm{MPa} \pm 0.15 \mathrm{MPa}$, $0.56 \mathrm{Mpa} \pm 0.15 \mathrm{MPa}$ and $2.24 \mathrm{MPa} \pm 0.72 \mathrm{MPa}$, respectively (Fig. 1c), which is consistent with the fact that dried foods are normally stiffer than the fresh ones [18, 19]. Similar in trend to the fresh peels, the modulus of dried TK is $\sim 3$ times higher than PSY and $\sim 4$ times higher than STY. The differences of elastic modulus of fresh pomelo peels are related to the dominant components, water and cellulose [20]. The water content within each variety of pomelo is uniform across the entire peel (Fig. 1d). On the other hand, the water contents are different among the three varieties. For example, the water content of PSY $(81.9 \% \pm 0.3 \%)$ is larger than that of STY $(79.9 \% \pm 0.4 \%)$ and TK $(74.2 \% \pm 0.2 \%)$ and the difference is statistically significant $(p<0.001)$. Upon the removing of lignin and hemicellulose from pomelo peels [16], the mean content of cellulose of TK is $33.1 \mathrm{~kg} / \mathrm{m}^{3}$, which is more than two times the cellulose found in PSY $\left(15.3 \mathrm{~kg} / \mathrm{m}^{3}\right)$ and STY $\left(14.7 \mathrm{~kg} / \mathrm{m}^{3}\right)$ (Fig. 1e); the trend in cellulose is consistent with the trend in modulus values.

The DIC technique was implemented to quantitatively understand the deformation under compression (Fig. 2). To explain the local strains, we use the term "parallel" for the direction of applied strain and the term "perpendicular" for the lateral direction. STY was taken as the example to illustrate the deformation behaviors. Upon the application of a compressive stress ( $2 \mathrm{kPa}$, global strain $4 \%$ ), the middle part immediately started to compress in both the parallel (Fig. 2b) and perpendicular (Fig. 2c) directions, while no obvious deformation was visible in the outer part. However, the local strains in the perpendicular direction were 
(a)
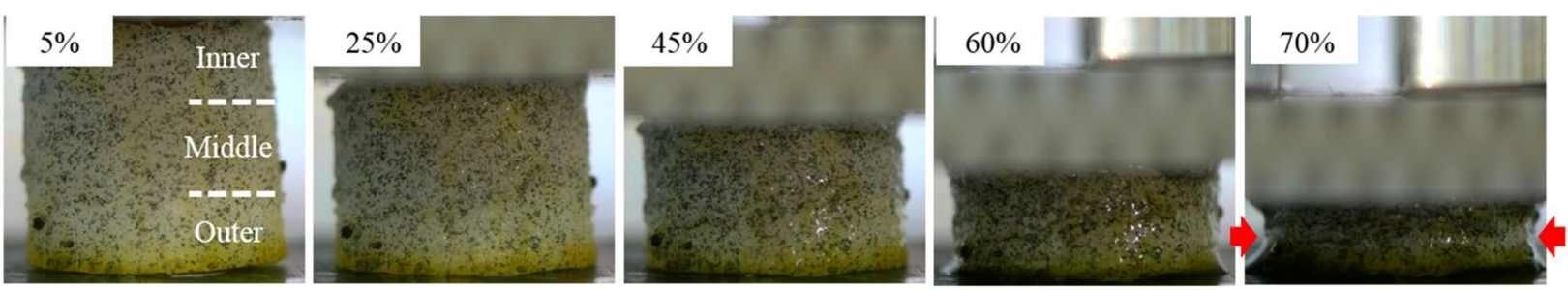

(b)

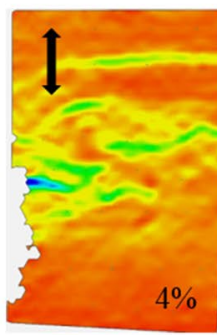

(c)
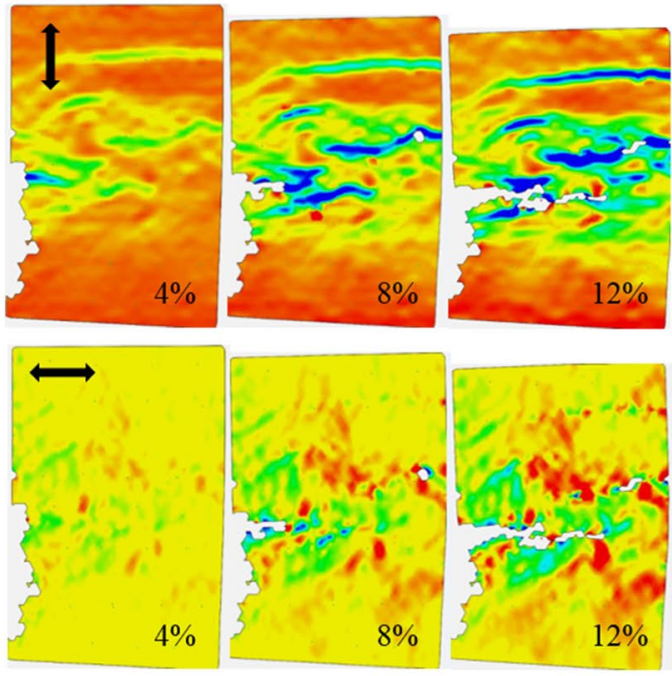
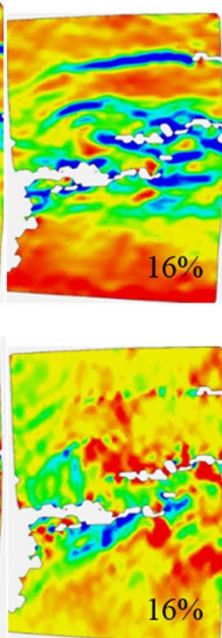

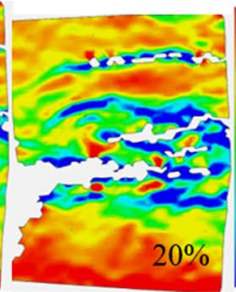

(d)

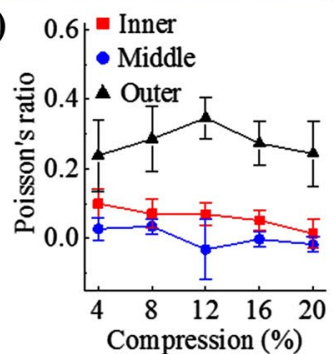

(e)

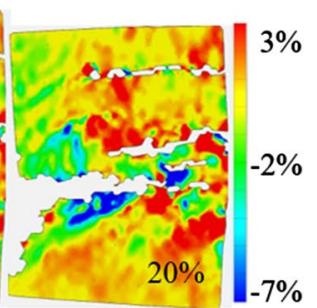

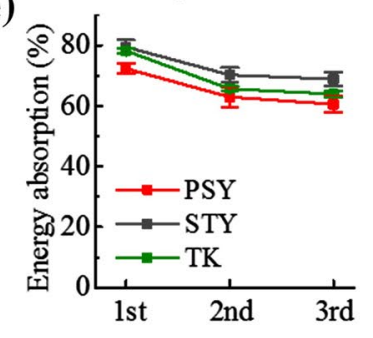

Fig. 2 Deformation of pomelo peels under quasi-static compression monitored by Digital Image Correlation (DIC). a Typical quasi-static compression process of STY with compression up to $70 \%$ strain. b and $\mathbf{c}$ Strain maps of compression strains of STY pomelo peel in the

much smaller than in the parallel direction. To get an idea of the parallel and perpendicular strains during compression, we calculated the Poisson's ratio for the three evenly distributed sections. Poisson's ratio is defined as the ratio of perpendicular strain (expansion is positive and contraction is negative) to parallel strain (compression is positive and elongation is negative), which gives a value around zero for the middle region (ranging between -0.032 and 0.035 , mean value of 0.025 ) (Fig. 2d). When the pressure exceeded $4 \mathrm{kPa}$ (global strain 12\%), local compressive strains in the parallel direction emerge in the inner part. However, no clear deformation is detected in the perpendicular direction; this ultimately leads to a Poisson's ratio fluctuating between 0.010 and 0.071 (mean value of 0.051 ) for the inner region (Fig. 2d). Therefore, once a pomelo is subjected to an impact, negligible lateral expansion occurs in the middle and inner regions, which can reduce internal stress concentrations. Moreover, the near-zero Poisson's ratio may allow the porous solid to densify in the middle region under pressure and prevent the further compression of the peel.

In contrast to the middle and inner parts, the outer part of the peel has a much higher resistance to compression. Almost no parallel deformation was detected in the outer perpendicular (b) and horizontal (c) direction, respectively. d Poisson's ratio of different parts of the peel in STY. e Energy absorption of fresh peels from three varieties of pomelos during quasi-static cyclic compression with a strain of $40 \%$. Values are means \pm s.d

region below a compressive stress of $6 \mathrm{kPa}$ (global strain $20 \%$ ). Hence, the low deformation of the outer part may serve as the first protective barrier for a pomelo to resist damage upon impact. When the stress exceeds $4 \mathrm{kPa}$ (global strain 12\%), slight expansion occurs in the perpendicular direction, yielding a positive Poisson's ratio in the outer part between 0.239 and 0.346 (mean value of 0.278 ), which allows the outer part to expand during compression and may ultimately result in the formation of a crack, if sufficient force is applied. Upon further compression over $45 \%$, wrinkles formed in the middle part and some liquid was squeezed out from the peel. As the strain approached $60 \%$, the inner and outer parts began to expand laterally, while no obvious lateral expansion was observed in the middle, forming the shape of a narrowing neck (Movs. 1). Similar deformation behaviors were found in PSY and TK (Fig. 3).

The capability of energy absorption, defined as the area in the stress-strain curve of a successive loading and unloading compression tests, was measured and compared among the three varieties of pomelo peels. On the first cycle, the amounts of absorbed energy was $79.5 \% \pm 2.3 \%$ for ST, which slightly reduced to $68.9 \% \pm 2.3 \%$ on the third cycle (Fig. 2e). Quite similar energy absorptions were also detected in PSY and TK during the loading-unloading 
(a)

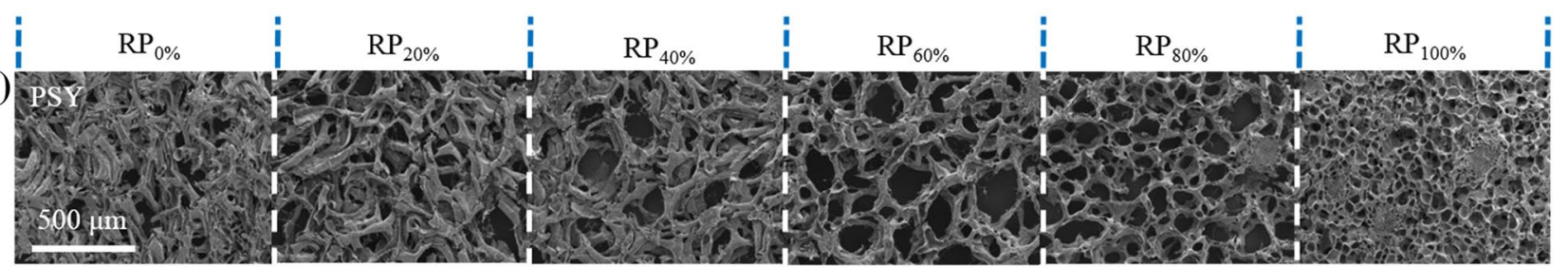

(b)

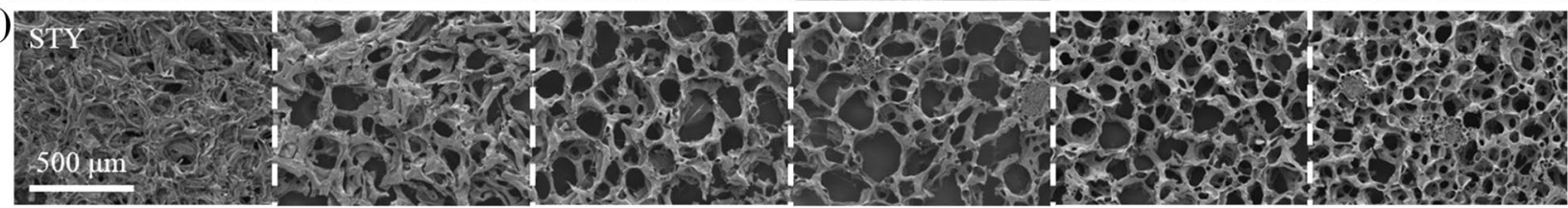

(c)

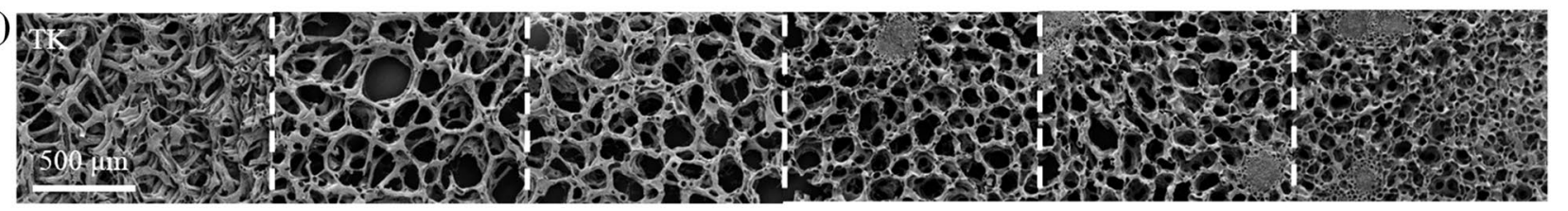

Fig. 3 Porous structure in pomelo peels revealed by SEM. Pomelo peels are equally divided into six parts from endocarp to exocarp, and each part is marked by the relative position (RP) away from the pulp.

cycles (Fig. 4). Under a compressive stress, the struts in a fresh pomelo peel bend or buckle, which causes cells to rupture and release fluids to the voids $[6,21]$. The cell rupture, the liquid flow, and the friction between the liquid and cells absorb part of the input energy. The deformation of struts and the loss of liquid reduce the energy absorption in the following loading/unloading cycles.

While the contents of water and cellulose in the three pomelo varieties, which have strong influence on the mechanical properties of pomelo peel, are different (Fig. 1), the deformation characteristics and the energy absorption ability among the three pomelo varieties are very close (Figs. 2 and 3). A question therefore emerges: What characteristics of pomelo peels leads to the similar capability in energy absorption in different varieties of pomelos? We hypothesize that it may result from the porous nature of the peels.

\subsection{Porous Structure in Pomelo Peels}

The porous structures of the peels in PSY, STY, and TK as a function of the position along the thickness were characterized in detail. The height and diameter of cylinder samples shrunk by $4 \%$ and $10 \%$ after drying, respectively, which are similar to the previous report [6]. The pomelo peel was evenly divided into six layers, which were marked as a percentage of the $\mathrm{RP}_{x \%}$ along the peel thickness from the endocarp $\left(\mathrm{RP}_{0 \%}\right)$ to the exocarp $\left(\mathrm{RP}_{100 \%}\right)$, and examined by scanning electron microscopy (SEM) (Fig. 3). While randomly dispersed vascular bundles are clearly visible between a-c SEM images of pomelo peels of PSY (a), STY (b) and TK (c) from endocarp $\left(\mathrm{RP}_{0 \%}\right)$ to exocarp $\left(\mathrm{RP}_{100 \%}\right)$

$\mathrm{RP}_{80 \%}$ and $\mathrm{RP}_{100 \%}$ in PSY, they were found between $\mathrm{RP}_{60 \%}$ and $\mathrm{RP}_{100 \%}$ in the other two varieties of pomelos. The randomly dispersed vascular bundles may be responsible for the larger dispersion of Poisson's ratios of the outer layers than that in the middle and inner layers (Fig. 2d and Figs. 3e, f).

The distribution of pore radius $(R)$, pore density $(\rho)$, and porosity $(\sigma)$ along the peel thickness for the three different regions of a single fruit were carefully extracted, taking STY as the main example (Fig. 4a, c, e). It is worth mentioning that the vascular bundles were automatically considered as part of the porous structure. In the peel of the top part of a fruit, the mean $R$ is $23.5 \mu \mathrm{m}$ at $\mathrm{RP}_{0 \%}$, which increases to $31.3 \mu \mathrm{m}$ at $\mathrm{RP}_{40 \%}$, and then decreases to $20.1 \mu \mathrm{m}$ at $\mathrm{RP}_{100 \%}$. Comparing the different regions of the fruit at $\mathrm{RP}_{0 \%}$, the mean $R$ at the top part is slightly larger than the middle $(17.2 \mu \mathrm{m})$ and bottom $(19.6 \mu \mathrm{m})$ parts of the fruits, although the pores at $\mathrm{RP}_{100 \%}$ are quite similar (top, middle and bottom are 20.5, 21.2 and $19.3 \mu \mathrm{m}$, respectively). In general, $R$ is the largest at RP between 40 and $60 \%$. (It should be noted that the peaks of the fitted cures are slightly shifted because the box-and-whiskers plots are shifted within each RP going from red to blue to black.) For PSY, the top part of the fruit also has larger pores than the middle and bottom parts of the fruits, similar to STY (Figs. 5a). However, TK does not show much difference in pore size for the three regions of the fruit (Figs. 5b). Moreover, the RP where the largest pores are found (i.e., the peak of the curve) is closer to the exocarp for PSY and closer to the endocarp for TK. Although there is some variation in the RP of the maximum porosity for different varieties, the general trend is consistent across all 
Fig. 4 Structure parameters of pomelo peels along the thickness direction from endocarp $\left(\mathrm{RP}_{0 \%}\right)$ to exocarp $\left(\mathrm{RP}_{100 \%}\right)$. a-f Pore radius $(R)(\mathbf{a}, \mathbf{b})$, pore density $(\rho)(\mathbf{c}, \mathbf{d})$ and porosity $(\sigma)(\mathbf{e}, \mathbf{f})$ as a function of RP in different parts of STY (a, c, e) and in the three varieties of pomelos $(\mathbf{b}, \mathbf{d}, \mathbf{f})$, respectively. In (b), (d) and (f), the top, middle, bottom parts in each variety of pomelo are considered to be uniform. The box plots are shown with median (horizontal center line), average (center point), interquartile range (IQR) (box edges), $1.5 \times \mathrm{IQR}$ (whiskers) and maximum/minimum (*), respectively. The solid lines are fitted based on the average values (a)

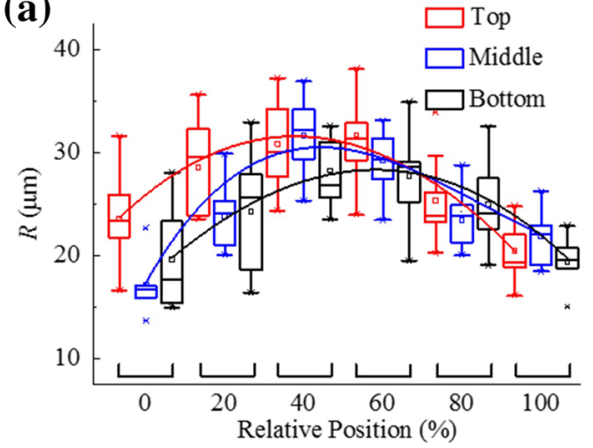

(c)
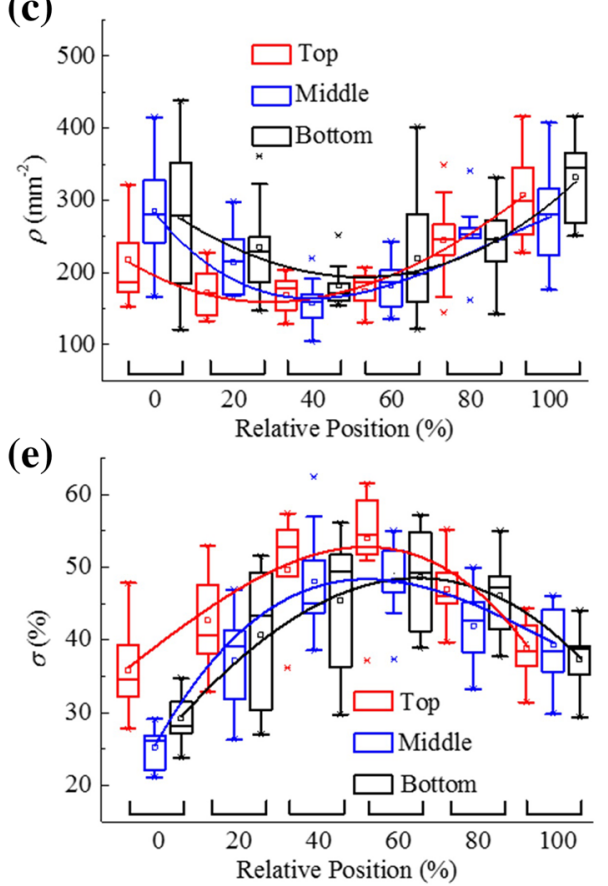
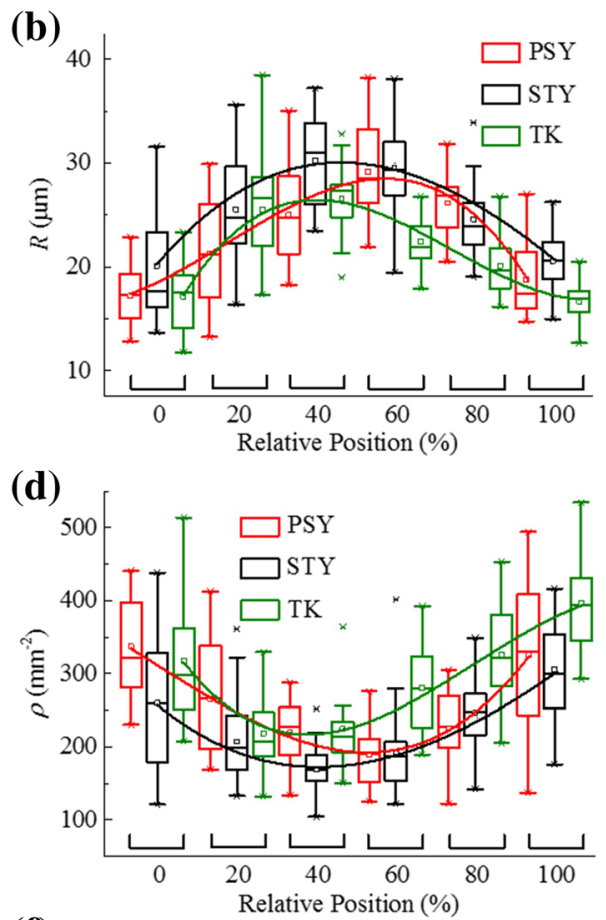

(f)

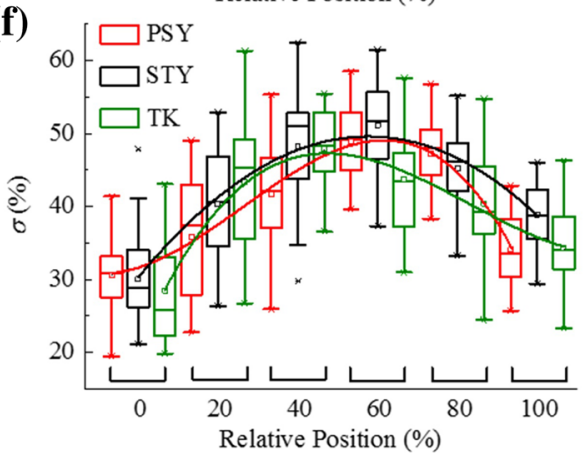

varieties; that is, the endocarp and the exocarp regions have smaller pores than the mesocarp region.

Since $R$ values along the thickness of pomelo peel within each variety are close at the parts of top, middle and bottom parts in a fruit, we take the mean value of the whole fruit to compare the different varieties (Fig. 4b). Generally, TK has the smallest pores, while STY has the largest pores. The maximum sized pores in PSY $(28.4 \mu \mathrm{m})$, STY $(29.6 \mu \mathrm{m})$ and TK $(26.6 \mu \mathrm{m})$ are located at the RP of $64 \%, 46 \%$ and $33 \%$, respectively.

The pore densities of the top, middle and bottom parts of the fruit were investigated, again taking STY as the main example (Fig. 4c). In the top part of the fruit, $\rho$ at $\mathrm{RP}_{0 \%}$ has a mean value of $217.7 \mathrm{~mm}^{-2}$. The pore densities at $\mathrm{RP}_{0 \%}$ in the middle and bottom parts are similar, having mean values of around 285.0 and $276.7 \mathrm{~mm}^{-2}$, respectively. $\rho$ decreases until it reaches a minimum value $\left(162.0 \mathrm{~mm}^{-2}\right)$ at $\mathrm{RP}_{35 \%}$, followed by an increase up to $309.5 \mathrm{~mm}^{-2}$ at $\mathrm{RP}_{100 \%}$, using the top part of STY as the example. A similar tendency was also observed in the middle and bottom parts of STY. Moreover, the same trends were found in PSY and TK (Figs. 5c and d). Since the pore densities at the top, middle and bottom parts of the fruit are similar within one variety, we averaged $\rho$ for the entire fruit to compare among varieties (Fig. 4d). In contrast to the pore radius, $\rho$ of TK is larger than PSY and STY. From the endocarp to the central mesocarp region, pore densities of PSY, STY and TK decrease; the minimum densities are $193.5,177.6$ and $215.6 \mathrm{~mm}^{-2}$ at RP of $59 \%, 40 \%$ and $31 \%$, respectively. At $\mathrm{RP}_{100 \%}$, pore densities of PSY, STY and TK are 325.4, 307.9 and $393.2 \mathrm{~mm}^{-2}$, respectively, which are the largest in each variety.

Combining the pore radius and density, the porosities of the peels of the three varieties were considered. For STY (Fig. 4e), the porosity of the top part is larger than the middle and bottom parts of the fruit. Along the thickness direction, the porosity increases from $36 \%$ at $\mathrm{RP}_{0 \%}$ to $60 \%$ at $\mathrm{RP}_{52 \%}$, followed by a decrease to $38 \%$ at $\mathrm{RP}_{100 \%}$. While the RP values of maximum porosity of the top and bottom parts of the fruit are almost identical, it is closer to the pulp in the middle part of the peel in STY. It explains why the 
(a)

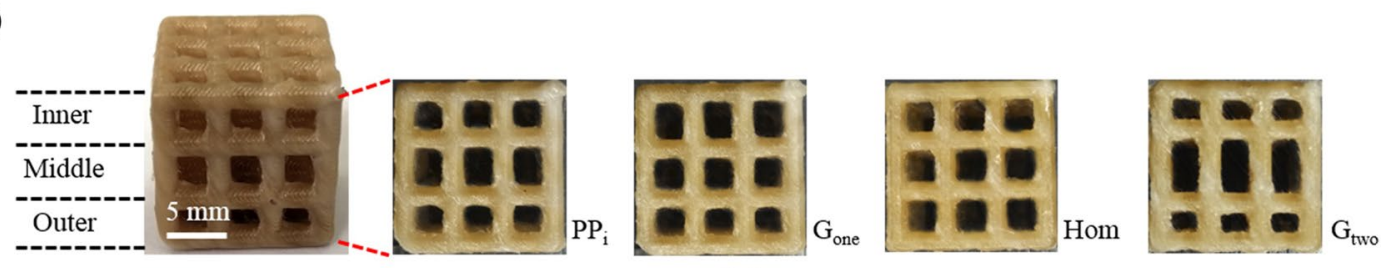

(b)

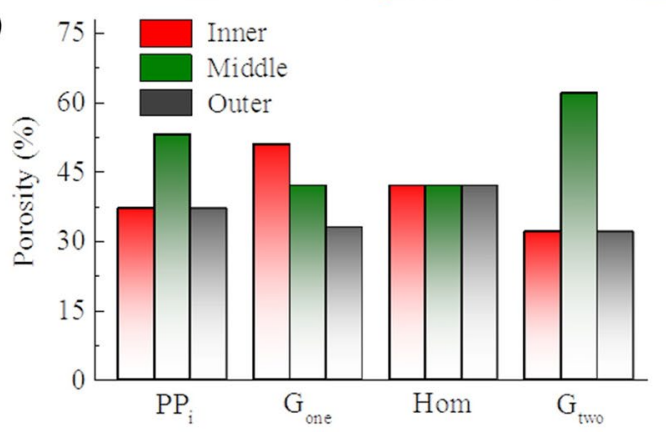

(d)

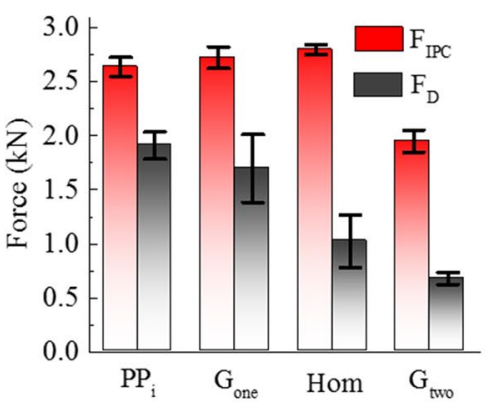

(e)

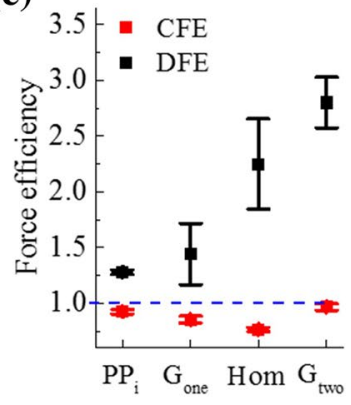

(c)

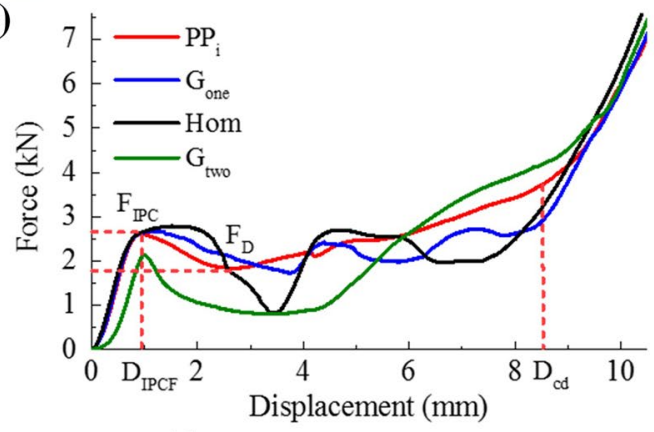

(f)

(g)

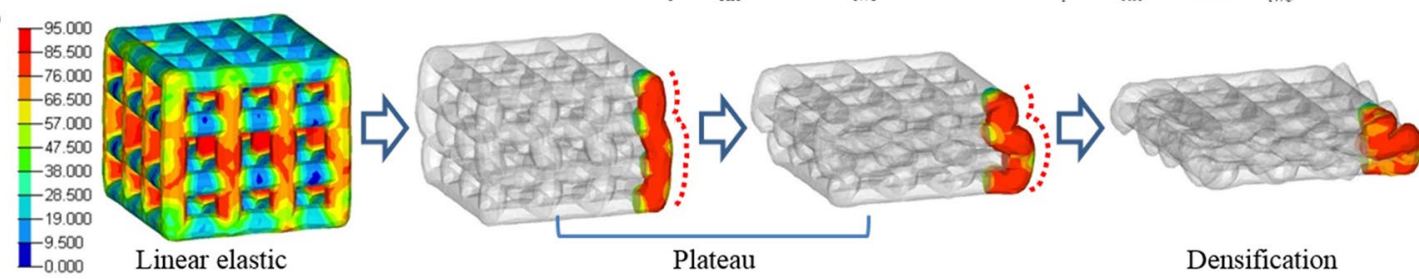

Fig. 5 Mechanical properties of additive manufactured polyetherether-ketone (PEEK) porous cubes. a, b Front view (a) and porosity (b) of four kinds of cubes, including the pomelo peel-inspired gradient cube $\left(\mathrm{PP}_{i}\right)$, one end gradient cube $\left(G_{\text {one }}\right)$, homogeneous cube (Hom) and two ends gradient cube $\left(G_{\mathrm{two}}\right)$. c Compression forcedisplacement curves with initial peak crushing force $\left(F_{\text {IPC }}\right)$, dropping force $\left(F_{\mathrm{D}}\right)$, compression distance of initial peak crushing force

deformation of pomelo peel first started in the middle part (Fig. 2) [17], which also agrees very well with the reported finite element simulations [22,23]. The large porosity in the middle part thus provides enough space for the deformed struts to collapse into, such that the lateral expansion does not occur in the middle part [4].

For PSY and TK, the RP values of maximum porosity are clearly different from that of STY (Figs. 5e and f), though the general trend remains the same (Fig. 4f). Taking the average across the entire fruit for each variety, the porosities of the three varieties are almost identical, showing a minimum value of $\sim 30 \%$ at $\mathrm{RP}_{0 \%}$, and a
$\left(D_{\text {IPCF }}\right)$ and critical displacement $\left(D_{\text {cd }}\right)$. d-f $\mathrm{F}_{\text {IPC }}$ and $F_{\mathrm{D}}(\mathbf{d})$, crush force efficiency (CFE) and dropping force efficiency (DFE) (e), critical strain $\left(\varepsilon_{\mathrm{cd}}\right)$ and specific energy absorption (SEA) (f) of $\mathrm{PP}_{i}, G_{\text {one }}$, Hom and $G_{\text {two }}$ during the compression, respectively. $\mathrm{g}$ Compressive stress distribution and deformation of simulated PEEK cubes of $\mathrm{PP}_{i}$. Values are means \pm s.d.

maximum porosity of around $49 \%$ in the middle of mesocarp (Fig. 4f). Therefore, the only difference among the three varieties is the RP of the maximum porosity, which are $68 \%, 57 \%$ and $41 \%$ for PSY, STY and TK, respectively.

\subsection{Pomelo Peel-Inspired Structures}

Inspired by the pore distribution in pomelo peels, PEEK cubes $\left(\mathrm{PP}_{\mathrm{i}}\right)$ were additively manufactured and compared to the non-pomelo peel-inspired structures which include: (1) a one end gradient cube $\left(G_{\text {one }}\right),(2)$ a homogeneous cube (Hom), and (3) both ends gradient cube $\left(G_{\text {two }}\right)$. For $\mathrm{PP}_{\mathrm{i}}$, the 
porosities going from the inner part to the outer part are $37 \%, 53 \%$ and $37 \%$ (Fig. 5a). The three other cubes have the following porosities from the inner to the outer parts (Fig. $5 b): G_{\text {one }}(51 \%, 42 \%$, and $33 \%)$, Hom ( $42 \%$ everywhere), and $G_{\mathrm{two}}(32 \%, 62 \%$, and $32 \%)$.

The quasi-static compression process of the 3D-printed structures were quantitatively investigated and compared. After the linear elastic deformation at small displacement, the first peak force in the compression process is defined as initial peak crushing force $\left(F_{\mathrm{IPC}}\right)$, and the corresponding displacement is $D_{\text {IPCF }}$ (Fig. 5c, d) [24]. Afterward, the compressive force comes to a plateau regime until the densification happens at a critical displacement $\left(D_{\mathrm{cd}}\right)$ (or the critical strain, $\varepsilon_{\mathrm{cd}}$ ) (Fig. 5c and Figs. 6). While the sizes of samples and pores are in the same order of magnitude, it will not affect $F_{\text {IPC }}$ and $\varepsilon_{\text {cd }}$, which has been demonstrated in openpore and closed-cell structures [25-27]. The minimum force detected just after the $F_{\text {IPC }}$ is defined as dropping force $\left(F_{\mathrm{D}}\right)$. The crush force efficiency (CFE) and the dropping force efficiency (DFE) (Fig. 5e), which are defined as the ratios of the mean compressive force between 0 and $D_{\text {cd }}$ to $F_{\text {IPC }}$ and $F_{\mathrm{D}}$, respectively [28], are used to indicate the fluctuation of force during the plateau regime. The closer the CFE (or DFE) to 1, the weaker is the fluctuation of force. In $\mathrm{PP}_{\mathrm{i}}$, $F_{\text {IPC }}=2.64 \pm 0.09 \mathrm{kN}$ and $F_{\mathrm{D}}=1.91 \pm 0.13 \mathrm{kN}$ resulted in a CFE of $0.925 \pm 0.021$ and a DFE of $1.279 \pm 0.020$ in $\mathrm{PP}_{\mathrm{i}}$ (Fig. 5d, e). The CFE and DFE of $\mathrm{PP}_{\mathrm{i}}$ are much closer to 1 as compared with the non-pomelo peel-inspired structures. It suggests a smooth and continuous deformation of the pomelo peel-inspired structure (Movs. 2). In contrast, abrupt changes in compression forces were detected in the nonpomelo peel-inspired structures (Movs. 3), which is not good for the object protection. Moreover, the $\varepsilon_{\mathrm{cd}}$ of $\mathrm{PP}_{i}(55 \pm 2 \%)$ is higher than that of Hom and $G_{\text {one }}$, but slightly lower than $G_{\text {two }}$ (Fig. 5f). The larger $\varepsilon_{\mathrm{cd}}$ and the weaker force fluctuation during compression thus result in a much higher SEA, which is defined as the absorbed energy per unit mass. The SEA of $\mathrm{PP}_{i}$ reached $12.94 \pm 0.45 \mathrm{~J} / \mathrm{g}$, which is $27 \%$ higher than that of $\operatorname{Hom}(10.21 \pm 0.89 \mathrm{~J} / \mathrm{g}), 9 \%$ and $19 \%$ higher than that of $G_{\text {one }}(11.92 \pm 1.05 \mathrm{~J} / \mathrm{g})$ and $G_{\text {two }}(10.89 \pm 0.79 \mathrm{~J} / \mathrm{g})$, respectively (Fig. 5f).

Numerical simulations of the deformation process are conducted to validate the compression of printed cubes. In the linear elastic stage, the stresses mainly concentrate in the struts along the direction of compression and distribute evenly in the inner, middle and outer parts of all the four kinds of cubes (Fig. 5g and Figs. 7). Once the stress exceeds the buckling strength at a displacement of $\sim 1.3 \mathrm{~mm}$, bending starts in the part of the largest porosity, which is the middle part in $\mathrm{PP}_{i}\left(G_{\mathrm{two}}\right)$ and the inner part in $G_{\text {one }}$ (Fig. 5g and Figs. 7, plateau stage). These results agree well with the quasi-static compression tests that show the collapse of pores starting in the middle part of a fresh pomelo peel (Fig. 2). It retards the deformation of the parts with smaller pores that is the inner and outer parts. The further compression condenses the larger pores, followed by the initiation of the deformation of the parts with smaller pores in $\operatorname{PP}_{i}\left(G_{\text {one }}\right.$ and $\left.G_{\text {two }}\right)$. This process greatly increases the duration of the plateau region of $\mathrm{PP}_{i}\left(G_{\text {one }}\right.$ and $\left.G_{\text {two }}\right)$ and delays the appearance of the final densification (Figs. 8). Meanwhile, the deformation in the larger pores does not reduce the compression strength. In contrast, simultaneous bending in the three parts occurs in Hom, which lasts continuously until the densification of the structure at a displacement of $8.9 \mathrm{~mm}$ (Figs. 8a). Therefore, with the higher compressive strength and the longer force plateau stage, $\mathrm{PP}_{i}$ has the highest energy absorption performance, which agrees well with the experimental results (Fig. $5 \mathrm{f}$ and Figs. 8b). These simulations, which are consistent with the experimental results, confirm the optimization of a porous structure through the natural evolution of pomelo peels for effective energy absorption.

\section{Conclusion}

In summary, we comprehensively quantified the structure and compressive response of the peels of three pomelo varieties. The structures of different varieties follow the same rule: the porosity is low near the endocarp and exocarp, and is highest in the mesocarp region. Moreover, the location having the maximum porosity seems to depend on the pomelo variety. Under quasi-static compression, the deformation starts within the region having the highest porosity, showing a Poisson's ratio close to zero. The region close to the exocarp stays less compressed and has a positive Poisson's ratio. The merits of the porous design extracted from pomelo peels for efficient energy absorption are demonstrated in additively manufactured porous PEEK cubes and numerical simulations. The results here offer a clear guidance to design lightweight materials with high energy absorption.

Supplementary Information The online version contains supplementary material available at https://doi.org/10.1007/s42235-021-00145-1.

Acknowledgements This work was supported by the National Key R\&D Program of China (2018YFB1105100) and National Natural Science Foundation of China (51973165).

Data Availability Data availability, the raw data required to reproduce these findings, are available from the corresponding author on reasonable request. 


\section{Declarations}

Conflict of Interests The authors declare that they have no conflict of interests.

Open Access This article is licensed under a Creative Commons Attribution 4.0 International License, which permits use, sharing, adaptation, distribution and reproduction in any medium or format, as long as you give appropriate credit to the original author(s) and the source, provide a link to the Creative Commons licence, and indicate if changes were made. The images or other third party material in this article are included in the article's Creative Commons licence, unless indicated otherwise in a credit line to the material. If material is not included in the article's Creative Commons licence and your intended use is not permitted by statutory regulation or exceeds the permitted use, you will need to obtain permission directly from the copyright holder. To view a copy of this licence, visit http://creativecommons.org/licenses/by/4.0/.

\section{References}

1. Zhang, Q., Yang, X., Li, P., Huang, G., \& Lu, T. J. (2015). Bioinspired engineering of honeycomb structure-using nature to inspire human innovation. Progress in Materials Science, 74, 332-400.

2. Chen, C. J., Kuang, Y. D., Zhu, S. Z., Burgert, I., Keplinger, T., Gong, A., Li, T., Berglund, L., Eichhorn, S. J., \& Hu, L. B. (2020). Structure-property-function relationships of natural and engineered wood. Nature Reviews Materials, 5, 642-666.

3. Zhang, L. L., Liu, Z. Q., Jiao, D., Zhang, J., Wang, S. G., Zhang, H., \& Zhang, Z. F. (2021). Natural cornstalk pith as an effective energy absorbing cellular material. Journal of Bionic Engineering, 18, 600-610.

4. Buhrig-Polaczek, A., Fleck, C., Speck, T., Schuler, P., Fischer, S. F., Caliaro, M., \& Thielen, M. (2016). Biomimetic cellular metalsusing hierarchical structuring for energy absorption. Bioinspiration \&amp; Biomimetics, 11, 045002.

5. Seidel, R., Thielen, M., Schmitt, C., Buhrig-Polaczek, A., Fleck, C., \& Speck, T. (2010). Fruit walls and nut shells as an inspiration for the design of bio-inspired impact resistant hierarchically structured materials. Design and Nature V: Comparing Design in Nature with Science and Engineering, Brebbia C, Carpi A, Eds., 138, 421-430.

6. Thielen, M., Speck, T., \& Seidel, R. (2015). Impact behaviour of freeze-dried and fresh pomelo (Citrus maxima) peel: influence of the hydration state. Royal Society Open Science, 2, 140322.

7. Ortiz, J., Zhang, G., \& McAdams, D. A. (2018). A model for the design of a pomelo peel bioinspired foam. Journal of Mechanical Design, 140, 114501

8. Fischer, S. F., Thielen, M., Loprang, R. R., Seidel, R., Fleck, C., Speck, T., \& Bührig-Polaczek, A. (2010). Pummelos as concept generators for biomimetically inspired low weight structures with excellent damping properties. Advanced Engineering Materials, 12, B658-B663.

9. Thielen, M., Schmitt, C. N., Eckert, S., Speck, T., \& Seidel, R. (2013). Structure-function relationship of the foam-like pomelo peel (Citrus maxima)-an inspiration for the development of biomimetic damping materials with high energy dissipation. Bioinspiration \&amp; Biomimetics, 8, 025001.

10. Thielen, M., Speck, T., \& Seidel, R. (2013). Viscoelasticity and compaction behaviour of the foam-like pomelo (Citrus maxima) peel. Journal of Materials Science, 48, 3469-3478.
11. Bhatnagar, A., Sillanpaa, M., \& Witek-Krowiak, A. (2015). Agricultural waste peels as versatile biomass for water purification-a review. Chemical Engineering Journal, 270, 244-271.

12. Ehsani, A., \& Parsimehr, H. (2020). Electrochemical energy storage electrodes via citrus fruits derived carbon: A minireview. Chemical Record, 20, 820-830.

13. Wang, B., Pan, B., \& Lubineau, G. (2017). Morphological evolution and internal strain mapping of pomelo peel using X-ray computed tomography and digital volume correlation. Materials \&amp; Design, 137, 305-315.

14. Zhang, W., Yin, S., Yu, T. X., \& Xu, J. (2019). Crushing resistance and energy absorption of pomelo peel inspired hierarchical honeycomb. International Journal of Impact Engineering, 125, 163-172.

15. Fischer, S. F., Thielen, M., Weiß, P., Seidel, R., Speck, T., BüHrigPolaczek, A., \& BüNck, M. (2014). Production and properties of a precision-cast bio-inspired composite. Journal of Materials Science, 49, 43-51.

16. Guan, H., Cheng, Z. Y., \& Wang, X. Q. (2018). Highly compressible wood sponges with a spring-like lamellar structure as effective and reusable oil absorbents. ACS Nano, 12, 10365-10373.

17. Li, Q. M., Magkiriadis, I., \& Harrigan, J. J. (2016). Compressive strain at the onset of densification of cellular solids. Journal of Cellular Plastics, 42, 371-392.

18. Kellogg, E. A., \& Bell, P. R. (1993). Green plants: Their origin and diversity. Journal of Ecology, 42, 16.

19. Telis, V. R. N., Telis-Romero, J., \& Gabas, A. L. (2005). Solids rheology for dehydrated food and biological materials. Drying Technology, 23, 759-780.

20. Liu, D., Song, J., Anderson, D. P., Chang, P. R., \& Hua, Y. (2012). Bamboo fiber and its reinforced composites: Structure and properties. Cellulose, 19, 1449-1480.

21. Zhu, H. X., \& Melrose, J. R. (2003). Time-dependent aspects of the mechanical properties of plant and vegetative tissues. Journal of Materials Science, 38, 2489-2498.

22. Shen, C. J., Lu, G., \& Yu, T. X. (2013). Dynamic behavior of graded honeycombs-a finite element study. Composite Structures, 98, 282-293.

23. Ajdari, A., Nayeb-Hashemi, H., \& Vaziri, A. (2011). Dynamic crushing and energy absorption of regular, irregular and functionally graded cellular structures. International Journal of Solids \&amp; Structures, 48, 506-516.

24. Ha, N. S., \& Lu, G. X. (2020). A review of recent research on bio-inspired structures and materials for energy absorption applications. Composites Part B-Engineering, 181, 107496.

25. Liu, Z. Q., Meyers, M. A., Zhang, Z. F., \& Ritchie, R. O. (2017). Functional gradients and heterogeneities in biological materials: Design principles, functions, and bioinspired applications. Progress in Materials Science, 88, 467-498.

26. Jin, T., Zhou, Z. W., Wang, Z. H., Wu, G. Y., \& Shu, X. F. (2015). Experimental study on the effects of specimen in-plane size on the mechanical behavior of aluminum hexagonal honeycombs. Materials Science and Engineering A, 635, 23-35.

27. Jeon, I., \& Asahina, T. (2005). The effect of structural defects on the compressive behavior of closed-cell Al foam. Acta Materialia, 53, 3415-3423.

28. Fang, J. G., Gao, Y. K., Sun, G. Y., Qiu, N., \& Li, Q. (2015). On design of multi-cell tubes under axial and oblique impact loads. Thin-walled Structures, 95, 115-126.

Publisher's Note Springer Nature remains neutral with regard to jurisdictional claims in published maps and institutional affiliations. 